\title{
Syndemic Health Disparities and Sexually Transmitted Infection Burden Among Black Men Who Have Sex with Men Engaged in Sex Work in the U.S.
}

\author{
Cristian J. Chandler ${ }^{1,2}$ (1) $\cdot$ Étienne Meunier ${ }^{3} \cdot$ Lisa A. Eaton $^{4} \cdot$ Elí Andrade $^{2,5} \cdot$ Leigh A. Bukowski $^{2,5}$. \\ Derrick D. Matthews ${ }^{6} \cdot$ Henry F. Raymond ${ }^{7} \cdot$ Ronald D. Stall ${ }^{2,5} \cdot$ M. Reuel Friedman $^{1,2}$
}

Received: 22 March 2020 / Revised: 17 July 2020 / Accepted: 21 August 2020 / Published online: 6 November 2020

\begin{abstract}
Black men who have sex with men (MSM) engaged in sex work (BMSM-SW) experience elevated HIV and sexually transmitted infection (STI) prevalence. Further, BMSM-SW have been shown to have higher rates of syndemic psychosocial health conditions which contribute to HIV risk behavior and incidence, and poorer care outcomes than other groups of men who have sex with men. However, syndemic perspectives have not been applied to understanding past-year STI burden among BMSM-SW in the U.S. Sexually active Black MSM $\geq 18$ years old were recruited from Black Pride events in six U.S. cities $(n=4421)$ between 2014 and 2017. Multivariable logistic regressions assessed correlates of past-year sex work engagement; whether BMSM-SW had higher odds of syndemic conditions; and whether BMSM-SW had higher odds of self-reported, past-year STI diagnoses. Structural equation models assessed relationships between sex work engagement, syndemic conditions, and STI controlled for sociodemographics and number of sexual partners. A total of 254 (5.7\%) Black MSM reported past-year sex work, of whom $45.3 \%$ were HIV positive. BMSM-SW were significantly more likely to be Hispanic, to report past-year bisexual behavior, and to report annual income $<\$ 10,000$. In multivariable models, BMSM-SW were significantly more likely to report intimate partner violence, assault victimization, polydrug use, and depression symptoms; they were also more likely to report past-year gonorrhea, chlamydia, and syphilis. Syndemic conditions mediated the relationship between past-year sex work and past-year STI burden, constituting a significant indirect effect. BMSM-SW in the U.S. face severe biopsychosocial health disparities. Interventions developed for BMSM engaged in sex work are lacking. Our results suggest that interventions containing safer sex work education and sex-positive biobehavioral HIV/STI prevention alongside substance use, mental health, employment, and education components will be most effective.
\end{abstract}

Keywords Sex work $\cdot$ Men who have sex with men · Sexually transmitted infections $\cdot$ Syndemics $\cdot$ Polysubstance use $\cdot$ Sexual orientation

Cristian J. Chandler

CJC143@pitt.edu

1 Department of Infectious Diseases and Microbiology, Graduate School of Public Health, University of Pittsburgh, 130 De Soto Street, Pittsburgh, PA 15261, USA

2 Center for LGBT Health Research, Graduate School of Public Health, University of Pittsburgh, Pittsburgh, PA, USA

3 Department of Sociomedical Sciences, Mailman School of Public Health, Columbia University, New York, NY, USA

4 Department of Human Development and Family Sciences, University of Connecticut, Storrs, CT, USA
5 Department of Behavioral and Community Health Sciences, Graduate School of Public Health, University of Pittsburgh, Pittsburgh, PA, USA

6 Department of Health Behavior, Gillings School of Global Public Health, University of North Carolina, Chapel Hill, NC, USA

7 Department of Biostatistics and Epidemiology, School of Public Health, Rutgers University, Piscataway, NJ, USA 


\section{Introduction}

Internationally, study findings vary widely regarding the health outcomes of men who have sex with men engaged in sex work (MSM-SW). "Sex work" is often used in the research literature as an umbrella term to refer to any form of exchange of sex for money, drugs, or other goods, also referred to as "transactional sex" (Bobashev, Zule, Osilla, Kline, \& Wechsberg, 2009) or "exchange sex" (Nerlander et al., 2017). A challenge in studying MSM-SW is that men who exchange sex often do not identify as sex workers or perceive their behavior as sex work and use locally specific terms or vernacular to refer to the practice (Baral et al., 2015; Koken, Bimbi, \& Parsons, 2010; Wong et al., 2008). Male sex workers mostly have male clientele, and the practice might have been obscured in general research on MSM (Baral et al., 2015). Further, male sex work is a very diverse practice happening through a multitude of online and physical venues, and involving individuals with various motivations and experiences (Minichiello, Scott, \& Callander, 2013; Schrimshaw, Siegel, \& Meunier, 2017). As such, it is important to understand the unique needs for health and disease prevention among distinct groups of MSM-SW.

In the U.S., there are demonstrated HIV and sexually transmitted infection (STI) disparities affecting Black MSM (BMSM) (Matthews et al., 2016; Maulsby et al., 2014). The literature has documented more HIV and STI infections among BMSM compared to MSM of other ethnoracial groups (Kelley et al., 2015; Lanier \& Sutton, 2013; Maulsby et al., 2014). In examinations of STI and HIV risk, individual behavior, such as substance use with sex, polysubstance use, or condomless sex, do not appear to be the sole drivers of the current disparities among Black MSM (Kelley et al., 2012; Maulsby et al., 2014; Sullivan et al., 2014; Tobin, Yang, King, Latkin, \& Curriero, 2016). While individual behaviors do not fully explain differences in HIV and STI rates, studies suggest that there is a complex confluence of factors, individual, dyadic, social and structural, that may better explain disparities (Maulsby et al., 2014; Sullivan et al., 2014). Examining sex work among BMSM could further our understanding of the HIV/STI disparities affecting this group.

The current literature only partly describes the connection between STI and sex work. In a survey of young MSM of color in New York City, having engaged in exchange sex was associated with being HIV positive and having been diagnosed with an STI in the prior 12 months (Patel, Masyukova, Sutton, \& Horvath, 2016). In a qualitative study, MSM who engaged in sex work reported less healthcare access and less STI testing than those who had not engaged in sex work (Underhill et al., 2014). Additionally, some surveys in the U.S. have shown Black MSM to be more likely to engage in sex work than those of other race/ethnicity (Bauermeister,
Eaton, \& Stephenson, 2016; Biello et al., 2017; Nerlander et al., 2017; Walters et al., 2020).

It has also been suggested that MSM of color may engage in risk behavior as a form of coping with interpersonal and structural stressors (Wilson et al., 2014). MSM who engage in sex work are more likely to report structural issues like low-income and housing insecurity (Bauermeister, Eaton, Meanley, Pingel, \& Partnership, 2017; Biello et al., 2017; Nerlander et al., 2017), lower educational achievements (Biello et al., 2017; Nerlander et al., 2017), or a history of incarceration (Nerlander et al., 2017; Philbin et al., 2018). Exchange sex and related sex work among MSM are also associated with psychosocial conditions of depression or anxiety (Bauermeister et al., 2016; Walters et al., 2020), hard drug use (Bauermeister et al., 2016; Biello et al., 2017; Nerlander et al., 2017; Walters et al., 2020), and having experienced harassment, assault, or intimate partner violence (Balaji et al., 2017; Walters et al., 2020). Sex work engagement and the related conditions require additional study compared to other MSM, particularly among Black MSM.

The theory of syndemic production has offered a method to study the impact of co-occurring conditions impacting health behavior and outcomes. The theory posits that the confluence of conditions synergizes to influence poorer health outcomes (Singer, Bulled, Ostrach, \& Mendenhall, 2017). Most often among MSM, syndemic theory has been used to predict HIV risk behavior or HIV/STI acquisition using psychosocial conditions in broad categories such as substance use, poor mental health and experiences of violence among other conditions (Stall, Friedman, \& Catania, 2008; Stall et al., 2003). Syndemic psychosocial health conditions have been attributed to HIV risk behavior, incidence, and HIV care outcomes in other groups of MSM (including those who identify as Black), and researchers have theorized that syndemic health conditions may operate similarly among men engaged in sex work (Biello, Colby, Closson, \& Mimiaga, 2014; Dyer et al., 2012; Mimiaga, Reisner, Tinsley, Mayer, \& Safren, 2009; Walters et al., 2020; Wilson et al., 2014). The literature has also noted the importance of syndemic theory in contemplating STI rates, particularly among people of color (Singer et al., 2006). However, it appears that little research has assessed the extent to which syndemic psychosocial health conditions may help explain STI burden among Black MSM engaged in sex work (BMSM-SW) in the U.S.

Drawing from other syndemic analyses, it is imperative to understand the biological and social forces that influence disparities in health outcomes (such as violence or homophobia), in order to confront efforts to pathologize marginalized groups, compounding the impact of these conditions (Farmer, 2001; Singer et al., 2006). Given that MSM-SW have been shown to have higher rates of syndemic psychosocial health conditions, and because syndemic psychosocial health conditions have been attributed to increased HIV and 
STI risk behavior, incidence, and poorer care outcomes in other groups of MSM, this analysis seeks to explore the relationship between past-year STI burden, experiences in sex work, and psychosocial comorbidities (a syndemic) among BMSM-SW.

\section{Method}

\section{Participants}

Promoting Our Worth, Equality and Resilience (POWER) a serial, cross-sectional survey of BMSM and transgender women collected data between 2014 and 2017 on $N=5858$ individuals. Data were collected across six cities (Atlanta, GA; Detroit, MI; Houston, TX; Memphis, TN; Philadelphia, PA; Washington, DC) using time location sampling (TLS) (Eaton et al., 2017; Friedman et al., 2018; Raymond \& McFarland, 2009). TLS was employed by randomly selecting two-hour blocks of time during official Black Pride events in each city where intercept zones were established, and potential participants were counted (Bukowski et al., 2018). Potential participants were approached and interested individuals were asked to screen for the study. Eligible participants were consented to a behavioral health survey and biological screening for HIV. Participants completed self-administered surveys via an audio computer-assisted self-interviewing assessment and, if they reported HIV negative or unknown serostatus, were offered rapid HIV antibody screening by local community-based organization or POWER study staff. Participants were compensated $\$ 10$ for a completed survey and $\$ 10$ for a completed HIV test. Further methods can be found elsewhere (Bukowski et al., 2018; Matthews et al., 2016).

Participants were included in the current analysis if they: (1) were assigned male sex at birth; (2) identified as male at the time of data collection; (3) were aged 18 and over; (4) reported anal sex with a man in the previous 12 months and (5) identified as Black, including multiracial participants. Data were screened for repeat assessments using a unique identifier code consisting of letters and numbers of easily recalled personal information (Dilley, McFarland, \& Kellogg, 2000; Hammer et al., 2003), and 301 duplicates were removed. The analytical sample resulted in $N=4421$.

\section{Measures}

\section{Sociodemographics}

Participants were asked the following sociodemographic variables: ethnicity: Hispanic ethnicity; city: city where survey and screening were completed; year: year of the study survey; age: age in years used continuously; annual income: dichotomized at the federal poverty level; education level: categorized as participants who never attended school, those who attended some school up to eighth grade, those who attended some high school, those who were high school/GED graduates, those with some college education, those with bachelor's degrees and those with any postgraduate education; and sexual identity: categorized as gay/homosexual, heterosexual/straight, bisexual and other.

\section{HIV Status}

HIV status was assessed using a combination of self-report and biological screening data. Participants were asked a single question about their HIV status: "What was the result of your last HIV test?" Participants were classified as HIV positive if they reported positive test result, or if they received a positive HIV test result during onsite screening. Participants who reported being HIV negative or unknown status were offered biological HIV specimen screening to verify their status. HIV tests included OraQuick (OraSure Technologies, Inc., Bethlehem, PA), Clearview STAT-PAK (Alere Inc., Waltham, MA), and INSTI (bioLytical Laboratories, Richmond, BC) tests.

\section{Past-Year Sex Work Engagement}

Past-year sex work engagement was identified in a two-step process comprised of six questions. Participants were asked: "In the past 12 months, did you ever give or take money, drugs or other goods for sex with a female partner?" with responses including "Yes," "No," "I don't know" and "Refuse to answer." Participants who answered "yes" to the preceding question were then asked if they had "received money, drugs or other goods for sex" or "gave money, drugs or other goods for sex" with female partners. Similarly, participants were asked: "In the past 12 months, did you ever give or take money, drugs or other goods for sex with a male partner?" with follow-up questions asking if they had "received money, drugs or other goods for sex" or "gave money, drugs or other goods for sex" with male partners. Responses were recorded dichotomously to reflect sex work engagement if participants reported that they had received money, drugs or other goods in exchange for sex within the previous year, regardless of the gender of the client. We consider these participants as Black MSM who engaged in sex work (BMSM-SW) based on their past-year behavior.

\section{Psychosocial Syndemic Comorbidities}

Syndemic comorbidities were assessed for dichotomous inclusion based on four types. Mental health proxy was assessed using depressive symptomology by scoring a total of 10 or more on the 10-item Centers for Epidemiological Study 
of Depression (CESD-10) (Andresen, Malmgren, Carter, \& Patrick, 1994). Polydrug use was assessed by reported experiences of using two or more substances at least monthly (amphetamines, crack cocaine, cocaine powder, GHB, heroin, inhalant "poppers", marijuana, MDMA/ecstasy or opioids not prescribed to the participant) in the previous three months (Friedman et al., 2018). Past-year intimate partner violence (IPV), and physical assault (i.e., hit, kicked, beat up or in any other way physically harmed) were also asked of participants as has been used in other syndemic analyses (Friedman et al., 2019). For structural equation modeling, a latent variable ("syndemic psychosocial conditions") comprised of each of these four observed psychosocial variables was created.

\section{Sexually Transmitted Infections}

Participants were asked if they had been diagnosed by a healthcare professional with each of the following STI within the previous year: (1) chlamydia, (2) gonorrhea, (3) syphilis, or (4) any other STI. STI were examined independently, and then as aggregate data to show any past-year STI and to demarcate three or more past-year STI. For structural equation modeling, a latent variable (“STI burden") comprised of each of the four reported STI variables was created.

\section{Statistical Analysis}

All analyses were completed using Stata version 14SE. Participants who selected that they refused to answer or were unsure of answers to survey questions were recorded as missing. For bivariate analyses of differences between Black MSW and other Black MSM, chi-square tests of difference were used to explore categorical sociodemographic variables and t-tests were used to describe difference by age. Multivariable logistic regression analyses were used to compare Black MSW and other BMSM on psychosocial variables, adjusting for sociodemographics and reported number of past-year sexual partners. Lastly, a mediation analysis using a structural equation model (SEM) was conducted to assess whether the relationship of sex work and past-year STI burden was mediated by psychosocial syndemic conditions. The SEM was conducted using maximum likelihood estimation (SEMML) with an observed information matrix (OIM) controlling for effects of covariates (Hispanic/Latino ethnicity; city; year sampled; HIV positive status; age 40 or older; low-income status; bisexual behavior; and number of past-year sexual partners) on both the mediator and outcome variables. Model fit for SEM was assessed via standardized root mean residuals (SRMR), assuming acceptable model fit at SRMR $<.08$; the model's $\chi^{2}$, root mean squared error of approximation (RMSEA) and comparative fit index (CFI) were also assessed. Syndemic models have generally viewed syndemic health conditions as predictors of, or explanatory factors for, HIV/STI incidence and care outcomes (Dyer et al., 2012; Dyer et al., 2020; Friedman et al., 2019; Guadamuz et al., 2013; Halkitis et al., 2015; Parsons, Grov, \& Golub, 2012; Tsai \& Burns, 2015). This conceptual framework provides our rationale for this mediation analysis, wherein we consider syndemic psychosocial conditions as factors that explain, or mediate, the relationship between sex work engagement and STI burden. Given that this relationship may be reversed, we also constructed an alternative SEM that models the mediating effect of STI burden on the relationship between sex work engagement and syndemic psychosocial health.

\section{Results}

Table 1 shows that, of the 4421 BMSM in the sample, 5.7\% $(n=254)$ reported sex work in the previous year. Compared to BMSM who did not report sex work, BMSM-SW were significantly more likely to report Hispanic/Latino ethnicity $\left(\chi^{2}=9.7, p<.01\right)$, annual income below Federal Poverty Level $\left(\chi^{2}=9.7, p<.001\right)$, and past-year bisexual behavior $\left(\chi^{2}=117.37, p<.001\right)$. There were significant differences between BMSM-SW and other BMSM in educational attainment $\left(\chi^{2}=103.77 ; p<.001\right)$, with BMSM-SW reporting lower rates of college or graduate degrees; sexual identity $\left(\chi^{2}=45.56, p<.001\right)$, with BMSM-SW reporting higher rates of bisexual identity; and HIV positive status $\left(\chi^{2}=5.34\right.$; $p<.05)$, with higher proportions of HIV positive status among BMSM-SW (39.8\% vs. $32.8 \%$ ). Compared with other BMSM, BMSM-SW had higher mean ages (32.2 vs. 30.5; $t=2.39, p<.05)$ and higher mean number of past-year sexual partners ( 16.8 vs. $5.4 ; t=2.78 ; p<.01)$. There were also significant differences between BMSM-SW and other BMSM in city of survey administration $\left(\chi^{2}=44.79, p<.001\right)$ and year of survey administration $\left(\chi^{2}=10.15, p<.05\right)$.

Table 2 shows that, in a multivariable regression analysis, past-year sex work was significantly associated with past-year bisexual behavior (aOR 3.37; 95\% CI 2.44, 4.66), annual income below Federal Poverty Level (aOR 2.72; 95\% CI 2.02, 3.66), and Hispanic/Latino ethnicity (aOR 2.16; $95 \%$ CI 1.21, 3.86). Bisexual identity, age over 40, HIV positive status, and city and year sampled were not significantly associated with past-year sex work in this multivariable model.

Table 3 demonstrates associations between past-year sex work engagement (predictor), psychosocial syndemic conditions, and past-year STI diagnoses (outcomes), in multiple multivariable logistic regressions adjusted for sociodemographics. Models with psychosocial variable outcomes were adjusted for city and year sampled, age 40 or older, Hispanic/ Latino ethnicity, bisexual behavior, HIV positive status, and annual income $<\$ 10,000$. Models with past-year STI diagnosis outcomes additionally adjusted for number of past-year 
Table 1 Bivariate associations between sociodemographics and sex work engagement (MSW) among Black MSM in POWER, 2014-2017 $(n=4421)$

\begin{tabular}{|c|c|c|c|c|}
\hline Sociodemographics & Subcategory & MSW (254) & Other MSM (4167) & Chi-square or $t$ test \\
\hline \multirow[t]{3}{*}{ Ethnicity } & & & & $9.70^{* *}$ \\
\hline & Hispanic/Latino & $17(6.7 \%)$ & $129(3.1 \%)$ & \\
\hline & Not Hispanic/Latino & $237(93.3 \%)$ & $4038(96.9 \%)$ & \\
\hline \multirow[t]{7}{*}{ City } & & & & $44.79 * * *$ \\
\hline & Philadelphia & $27(10.6 \%)$ & $591(14.2 \%)$ & \\
\hline & Houston & $59(23.2 \%)$ & $907(21.8 \%)$ & \\
\hline & Washington, D.C. & $30(11.8 \%)$ & $909(21.8 \%)$ & \\
\hline & Detroit & $60(23.6 \%)$ & $468(11.2 \%)$ & \\
\hline & Atlanta & $75(29.5 \%)$ & $1223(29.3 \%)$ & \\
\hline & Memphis & $3(1.2 \%)$ & $69(1.7 \%)$ & \\
\hline \multirow[t]{5}{*}{ Year } & & & & $10.15^{*}$ \\
\hline & 2014 & $82(32.3 \%)$ & $1217(29.2 \%)$ & \\
\hline & 2015 & $73(28.7 \%)$ & $1419(34.1 \%)$ & \\
\hline & 2016 & $58(22.8 \%)$ & $1085(26.0 \%)$ & \\
\hline & 2017 & $41(16.1 \%)$ & $446(10.7 \%)$ & \\
\hline Age & Mean age & 32.2 & 30.5 & $t=2.39 *$ \\
\hline \multirow[t]{2}{*}{ Annual income } & & & & $110.20 * * *$ \\
\hline & Below FPL & $113(45.0 \%)$ & $738(18.0 \%)$ & \\
\hline Sexual partners & $\begin{array}{l}\text { Number of total past-year } \\
\text { sexual partners }\end{array}$ & 16.8 & 5.4 & $t=2.78^{* *}$ \\
\hline \multirow[t]{8}{*}{ Education } & & & & $103.77 * * *$ \\
\hline & Never attended school & $14(5.6 \%)$ & $100(2.4 \%)$ & \\
\hline & 1st-8th grade & $13(5.2 \%)$ & $55(1.3 \%)$ & \\
\hline & 9th-11th grade & $24(9.5 \%)$ & $94(2.3 \%)$ & \\
\hline & 12th grade or GED & $69(27.4 \%)$ & $817(19.8 \%)$ & \\
\hline & Some college/A.D. & $79(31.3 \%)$ & $1533(37.1 \%)$ & \\
\hline & Bachelor's degree & $37(14.7 \%)$ & $1018(24.6 \%)$ & \\
\hline & Any postgraduate studies & $16(6.3 \%)$ & $510(12.3 \%)$ & \\
\hline \multirow[t]{4}{*}{ HIV Status } & & & & $5.34 *$ \\
\hline & Negative & $122(48.0 \%)$ & $2273(54.5 \%)$ & \\
\hline & Positive & $101(39.8 \%)$ & $1367(32.8 \%)$ & \\
\hline & Unknown/untested/missing & $31(12.2 \%)$ & $527(12.6 \%)$ & \\
\hline \multirow[t]{5}{*}{ Sexual identity } & & & & $45.56 * * *$ \\
\hline & Gay/Same Gender Loving & $172(67.7 \%)$ & $3416(82.0 \%)$ & \\
\hline & Heterosexual or "straight" & $8(3.1 \%)$ & $29(0.7 \%)$ & \\
\hline & Bisexual & $72(28.3 \%)$ & $667(16.0 \%)$ & \\
\hline & Other & $2(0.8 \%)$ & $55(1.3 \%)$ & \\
\hline \multirow[t]{3}{*}{ Sexual behavior } & & & & $117.37 * * *$ \\
\hline & Past-year MSMO & $147(57.9 \%)$ & $3513(84.3 \%)$ & \\
\hline & Past-year MSMW & $107(42.1 \%)$ & $654(18.6 \%)$ & \\
\hline
\end{tabular}

$* p<.05 ; * * p<.01 ; * * * p<.001$ sexual partners. BMSM-SW were significantly more likely to report depressive symptoms (59.4\% vs. $22.2 \%$; aOR 3.88 , $95 \%$ CI $2.91,5.19)$; past 3 -month polydrug use (36.7\% vs. $4.2 \%$; aOR $8.42,95 \%$ CI $6.13,11.56$ ); past-year intimate partner violence ( $47.1 \%$ vs. $15.3 \%$; aOR $4.17,95 \%$ CI $3.11,5.60)$, and past-year physical assault (49.0\% vs. $12.6 \%$; aOR 5.35, $95 \%$ CI 3.97, 7.22). BMSM-SW were significantly more likely than other BMSM to report past-year diagnoses of gonorrhea (26.4\% vs. $11.8 \%$; aOR $2.06,95 \%$ CI 1.47, 2.89), chlamydia (24.0\% vs. $8.9 \%$; aOR $2.32,95 \%$ CI $1.63,3.31)$, syphilis (19.0\% vs. $7.9 \%$; aOR 1.97, 95\% CI 1.34, 2.88), and any other STI (16.9\% vs. $6.1 \%$; aOR $2.42,95 \%$ CI $1.62,3.64)$. Compared to other BMSM, BMSM-SW had significantly higher odds of reporting any past-year STI diagnoses (36.0\% vs. $18.1 \%$; aOR $2.00,95 \%$ CI $1.47,2.72$ ) and of reporting 
Table 2 Multivariable logistic regression model showing sociodemographic correlates of sex work engagement among sexually active Black MSM, 2014-2017 in the POWER study $(n=4421)$ : adjusted odds ratios $(\mathrm{aOR})$

\begin{tabular}{|c|c|c|c|c|c|}
\hline Sociodemographics & Subcategory & $\begin{array}{l}\text { Adjusted odds } \\
\text { ratio (aOR) }\end{array}$ & 95\% CI (lower) & 95\% CI (higher) & $p$ value \\
\hline Bisexual behavior & Past-year & 3.37 & 2.44 & 4.66 & $<.001$ \\
\hline Bisexual identity & Current & 1.12 & 0.79 & 1.59 & .529 \\
\hline Age 40 or older & & 1.15 & 0.80 & 1.66 & .441 \\
\hline \multicolumn{6}{|l|}{ City sampled } \\
\hline & Philadelphia & REF (1.00) & - & - & - \\
\hline & Houston & 1.53 & 0.90 & 2.58 & .115 \\
\hline & Washington, D.C. & 0.73 & 0.39 & 1.36 & .327 \\
\hline & Detroit & 1.68 & 0.96 & 2.94 & .070 \\
\hline & Atlanta & 1.39 & 0.83 & 2.31 & .207 \\
\hline & Memphis & 0.97 & 0.27 & 3.51 & .968 \\
\hline \multicolumn{6}{|l|}{ Year sampled } \\
\hline & 2014 & REF (1.00) & - & - & - \\
\hline & 2015 & 0.83 & 0.58 & 1.20 & .317 \\
\hline & 2016 & 0.71 & 0.48 & 1.05 & .086 \\
\hline & 2017 & 1.02 & 0.66 & 1.58 & .927 \\
\hline Income & Below FPL & 2.72 & 2.02 & 3.66 & $<.001$ \\
\hline Hispanic/Latino & & 2.16 & 1.21 & 3.86 & .009 \\
\hline HIV positive & & 1.28 & 0.97 & 1.71 & .086 \\
\hline
\end{tabular}

Categories with $p$ values $<.05$ denoted in bold

\begin{tabular}{lllll}
\hline Outcome & Subcategory & MSW (245) & Other MSM (4176) & AOR (95\% CI) \\
\hline $\begin{array}{c}\text { Psychosocial } \\
\text { syndemic } \\
\text { conditions }\end{array}$ & Depression symptoms & $145(59.4 \%)$ & $921(22.2 \%)$ & $\mathbf{3 . 8 8}(\mathbf{2 . 9 1 , 5 . 1 9})$ \\
& Polydrug use, past 3 months & $90(36.7 \%)$ & $174(4.2 \%)$ & $\mathbf{8 . 4 2}(\mathbf{6 . 1 3}, \mathbf{1 1 . 5 6})$ \\
& $\begin{array}{l}\text { Intimate partner violence } \\
\text { (IPV), past year }\end{array}$ & $115(47.1 \%)$ & $638(15.3 \%)$ & $\mathbf{4 . 1 7}(\mathbf{3 . 1 1}, \mathbf{5 . 6 0})$ \\
& Physical assault, past year & $119(49.0 \%)$ & $525(12.6 \%)$ & $\mathbf{5 . 3 5}(\mathbf{3 . 9 7}, \mathbf{7 . 2 2})$ \\
Past-year STI & Gonorrhea & $64(26.4 \%)$ & $486(11.8 \%)$ & $\mathbf{2 . 0 6}(\mathbf{1 . 4 7 , 2 . 8 9})$ \\
diagnosis & Chlamydia & $58(24.0 \%)$ & $370(8.9 \%)$ & $\mathbf{2 . 3 2}(\mathbf{1 . 6 3}, \mathbf{3 . 3 1})$ \\
& Syphilis & $46(19.0 \%)$ & $325(7.9 \%)$ & $\mathbf{1 . 9 7}(\mathbf{1 . 3 4 , 2 . 8 8})$ \\
& Any other STI & $41(16.9 \%)$ & $252(6.1 \%)$ & $\mathbf{2 . 4 2}(\mathbf{1 . 6 2 , 3 . 6 4 )}$ \\
& Any STI diagnosis & $87(36.0 \%)$ & $748(18.1 \%)$ & $\mathbf{2 . 0 0}(\mathbf{1 . 4 7 , 2 . 7 2})$ \\
& 3 or more STI diagnoses & $37(15.3 \%)$ & $195(4.7 \%)$ & $\mathbf{2 . 4 5}(\mathbf{1 . 5 9 , 3 . 7 7})$ \\
\hline
\end{tabular}

Models with psychosocial variable outcomes were adjusted for city and year sampled, age $>39$, Hispanic/ Latino ethnicity, bisexual behavior, HIV positive status, and annual income $<\$ 10,000$. Models with pastyear STI diagnosis outcomes additionally adjusted for number of sexual partners

Bold aOR values represent significance at $p<.05$ three or more past-year STI diagnoses (15.3\% vs. $4.7 \%$; aOR $2.45,95 \%$ CI $1.59,3.77)$.

Figure 1 illustrates results from the structural equation model of the relationship between sex work engagement (predictor) and past-year STI burden (outcome), mediated by psychosocial syndemic factors. This SEM was adjusted for year, city, income, bisexual behavior, Hispanic ethnicity, HIV positive status, bisexual behavior, number of past-year sexual partners, and age $\geq 40$, on both the mediator and outcome variables. Model fit was considered good $(\mathrm{SRMR}=0.032$; RMSEA $=0.049 ;$ CFI $=0.928 ;$ TLI $=0.902$ ). Among
BMSM-SW, there were significant total effects between sex work engagement and past-year STI burden $(\beta=0.11 \pm 0.02$; $p<.001)$. There were also significant total effects between sex work engagement and psychosocial syndemic factors $(\beta=0.33 \pm 0.02, p<.001)$ and between psychosocial syndemic factors and past-year STI burden $(\beta=0.21 \pm 0.02$, $p<.001)$. Syndemic psychosocial factors constituted a significant indirect effect on the relationship between past-year sex work engagement and STI burden $(\beta=0.07 \pm 0.01, p<.001)$.

Table 4 shows the extent of partial mediation by syndemic psychosocial conditions of the relationship between sex work 


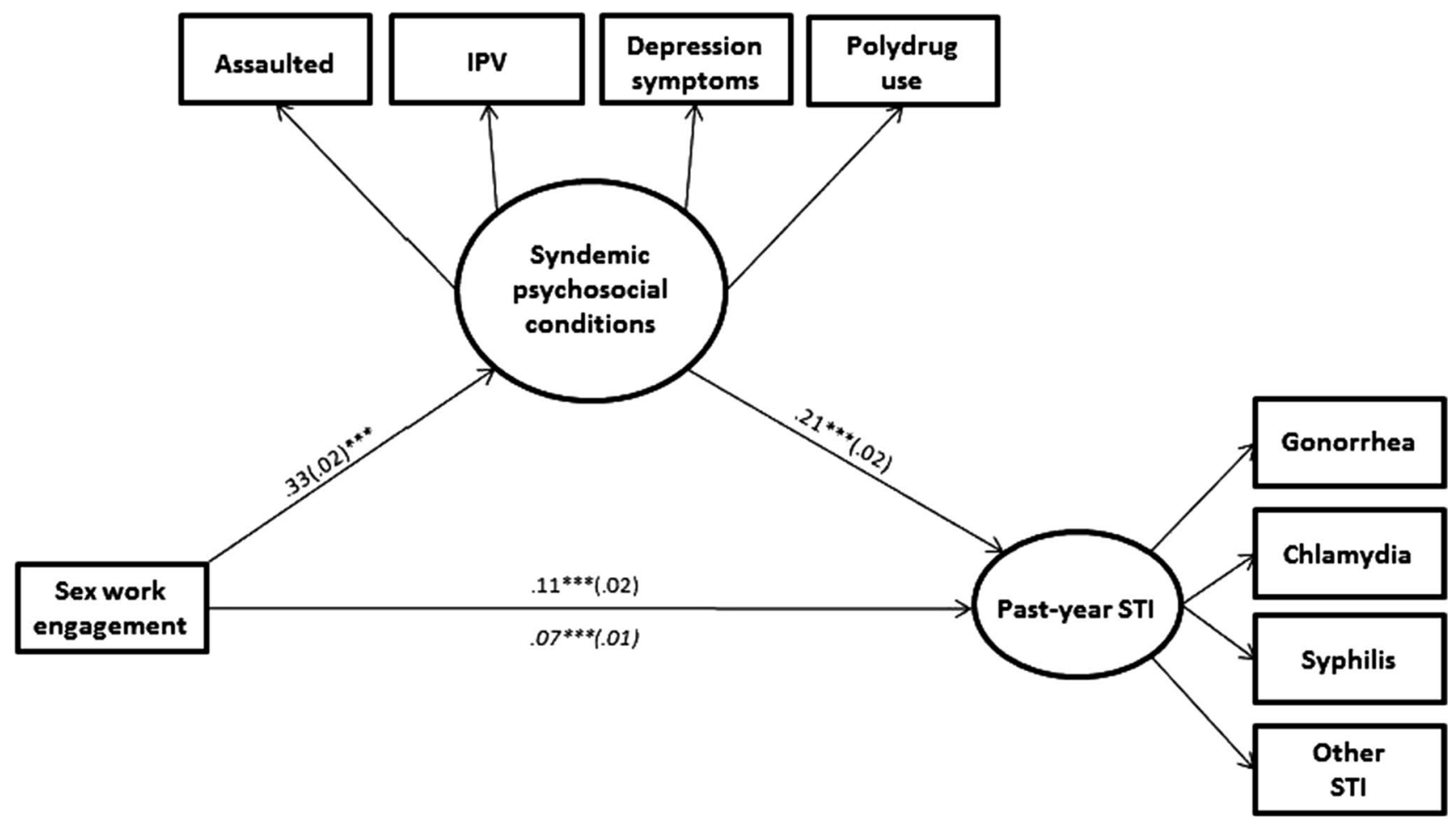

Fig. 1 Structural equation model showing total and indirect effects pathways between past-year sex work, syndemic psychosocial conditions, and STI burden among sexually active Black men in the POWER study, 2014-2017. *** $p<.001$. Path coefficients and standard errors (parenthesized) are shown for total effects pathways. Italicized path coefficients and standard errors (parenthesized) are shown

engagement and STI burden among BMSM-SW in the current sample. Syndemic psychosocial conditions were estimated to account for $64.2 \%$ (95\% CI $57.8 \%, 76.6 \%$ ) of this relationship. The full table of total, direct, and indirect effects from this SEM can be found in "Appendix 1".

"Appendix 2" shows results from the structural equation model of the relationship between sex work engagement for indirect effects pathways. Model adjusted for year, city, income, bisexual behavior, Hispanic ethnicity, HIV positive status, bisexual behavior, and age $\geq 40$. Covariate paths are suppressed for interpretability. Fit statistics are as follows: SRMR $=0.032$; RMSEA $=0.049$ (95\% CI 0.046, 0.052); CFI $=0.928 ; \mathrm{TLI}=0.902$

(predictor) and psychosocial health conditions (outcome), mediated by past-year STI burden. This SEM was adjusted for year, city, income, bisexual behavior, Hispanic ethnicity, HIV positive status, bisexual behavior, number of past-year sexual partners, and age $\geq 40$, on both the mediator and outcome variables. Model fit was considered $\operatorname{good}(\mathrm{SRMR}=0.034 ; \mathrm{RMSEA}=0.049 ; \mathrm{CFI}=0.928$;

Table 4 Total and indirect path coefficients and standard errors in pathways between sex work engagement and STI among Black MSM in the POWER study, 2014-2017 $(n=4421)$

\begin{tabular}{|c|c|c|c|c|c|}
\hline \multicolumn{6}{|c|}{ Pathways to past-year STI burden (latent variable) } \\
\hline \multirow[t]{2}{*}{ Predictors and covariates } & \multicolumn{2}{|l|}{ Total effects } & \multicolumn{3}{|c|}{$\begin{array}{l}\text { Indirect effects in model adjusting for mediator (latent syndemic psychosocial } \\
\text { conditions) }\end{array}$} \\
\hline & $\beta(\operatorname{SE} \beta)$ & $p$ & $\beta(\operatorname{SE} \beta)$ & $p$ & $\begin{array}{l}\text { Proportion of effect mediated by } \\
\text { syndemic psychosocial condi- } \\
\text { tions (\% and } 95 \% \mathrm{CI} \text { ) }\end{array}$ \\
\hline Past-year sex work engagement & $0.11(0.02)$ & $<.001$ & $0.07(0.01)$ & $<.001$ & $64.2 \%(57.8 \%, 76.6 \%)$ \\
\hline
\end{tabular}

Beta coefficients and respective standard errors reported

Model adjusted for year, city, income, bisexual behavior, Hispanic ethnicity, HIV positive status, bisexual behavior, number of past-year sexual partners, and age $\geq 40 . \mathrm{SRMR}=0.032 ; \mathrm{RMSEA}=0.049(95 \% \mathrm{CI} 0.046,0.052) ; \mathrm{CFI}=0.928 ; \mathrm{TLI}=0.902$ 
TLI $=0.902$ ). Among BMSM-SW, there were significant total effects between sex work engagement and syndemic psychosocial health $(\beta=0.33 \pm 0.02 ; p<.001)$. There were also significant total effects between sex work engagement and past-year STI burden $(\beta=0.11 \pm 0.02, p<.001)$ and between past-year STI burden and psychosocial syndemic ( $\beta=0.36 \pm 0.03, p<.001)$. Past-year STI burden constituted a significant indirect effect on the relationship between pastyear sex work engagement and psychosocial health syndemic $(\beta=0.04 \pm 0.01, p<.001)$.

"Appendix 3" shows the extent of partial mediation by syndemic psychosocial conditions of the relationship between sex work engagement and psychosocial health syndemic among BMSM-SW in the current sample. Syndemic psychosocial health conditions were estimated to account for $11.9 \%$ (95\% CI 8.9\%, 14.2\%) of this relationship.

\section{Discussion}

Our findings suggest that BMSM-SW experience a constellation of psychosocial syndemic comorbidities that contribute substantially to their disparity in past-year STI diagnoses over and above disparities seen in samples of other BMSM. Even in models controlling for number of sexual partners, BMSM-SW had higher odds of reporting all four psychosocial conditions. The comparative odds ratios for depression, physical violence, intimate partner violence, and polydrug use describe a group of BMSM-SW at the center of a syndemic requiring action. While some studies have noted that MSM-SW have elevated STI rates compared to other MSM (dos Ramos Farías et al., 2011; Mgbako et al., 2019; Muraguri et al., 2015; Patel et al., 2016), this study used a syndemic framework to contextualize the relationship between sex work engagement and STI burden.

Similar to previous studies, sex work was more likely among BMSM that reported annual income below $\$ 10,000$ as well as increased psychosocial conditions including polysubstance use, depressive symptomology, and IPV (Bauermeister et al., 2016; Walters et al., 2020). Given that: (1) the HIV epidemic among Black MSM has been attributed to higher background STI (Maulsby et al., 2014); (2) psychosocial conditions are associated with HIV/STI risk among Black MSM (T. P. Dyer et al., 2012; Mustanski et al., 2017); and (3) Black MSM have been more likely to report sex work engagement than men of other races and ethnicities (Bauermeister et al., 2017; Biello et al., 2017; Walters et al., 2020), the fact that BMSM-SW in this sample were significantly more likely to report psychosocial conditions and STI burden makes a comprehensive prevention approach imperative.

BMSM-SW in the U.S. face profound biopsychosocial health disparities, constituting a severe syndemic. These data augment findings of previous literature on MSM-SW in three ways: (1) this analysis supports previous literature offering insights into the mechanisms which contribute to elevated STI; (2) provides support to the use of psychosocial syndemics to discuss STI-related health outcomes; and (3) this analysis allowed for a deeper understanding of a psychosocial syndemic among Black MSM, rather than between Black MSM and MSM of ethnoracial groups. This novel analysis explores the analytical framework for the mediation of STI burden by psychosocial syndemic among BMSM-SW, an understudied population. Such findings warrant urgency in initiating responsible, respectful, and sex-positive harm reduction-based interventions and research for MSM-SW, particularly BMSM-SW.

These data must be evaluated in light of limitations. Data were serial cross-sectional, not allowing for causal associations, but may guide future research. For example, given what is known about how syndemics occur, are BMSM-SW more likely to report psychosocial conditions spurred by sex work engagement or are psychosocial conditions spurring sex work? Our models showed significant associations between sex work engagement, past-year STI burden, and a psychosocial syndemic. Moreover, we found significant mediation effects even when reversing the causal order: pastyear STI burden mediated the relationship between sex work engagement and psychosocial health syndemic, and psychosocial health syndemic mediated the relationship between sex work engagement and past-year STI burden. Because our data were cross-sectional, we could not conduct temporal mediation approaches that might otherwise disentangle the relationships between these intertwined variables. The SEM that we constructed was chosen because they are robust to concurrence of syndemic psychosocial conditions, accounting for differences in factor loadings that simple summative variables do not. However, our construction of latent variables in these models did not assess the effects of interaction between comprising variables, as Tsai and Burns (2015) have suggested, on relationships between predictors and outcomes. Although participants were asked about pastyear engagement in sex work, they were not asked about sex worker or escort identity; therefore, it is not known how these participants identify themselves. Relatedly, participants were not asked about employment in exchange sex or other forms of sex work, income specific to sex work, or the level of outness of their sex work engagement; future studies will benefit from including these variables. While most variables were self-reported by participants, HIV status was verified via HIV antibody screening by all consenting participants. Data analyzed included STI diagnoses in the previous year but did not assess access of STI screenings by participants. Age was dichotomized at age 40 as a covariate, and therefore information about age and the impact of age on this relationship is limited. These participants were encountered in national Black Pride events, which may indicate a social 
connectedness to the LGBT community not seen in other MSM, limiting the generalizability of this analysis; however, this analysis highlights the experience of a sizeable sample of BMSM-SW and other BMSM.

The psychosocial status of BMSM-SW in our analyses demonstrated the urgent need to develop and disseminate support interventions for sex work involvement, in addition to making space for the unique needs of BMSM-SW. These data suggest opportunities for intervention design, including better integration of STI screening in comprehensive sexual health services to include: substance use, mental health, employment, and education assistance concomitant with safer sex work education.

Studies have identified that some MSM engaged in sex work, particularly those of lower socioeconomic status, have challenges in negotiating condoms with clients who may offer a large premium for condomless sex (Baral et al., 2015; Edeza et al., 2020; Valente et al., 2020). Additionally, a study of MSM in Atlanta found that BMSM were more likely than White MSM to report condom failure or incomplete condom use linked to oil-based lubricants, erectile challenges or lack of condom fit (Hernández-Romieu, Siegler, Sullivan, Crosby, $\&$ Rosenberg, 2014). Among BMSM, differences in condom use have also been linked to gender role conflict among men who have sex with men and women, and internalized homophobia among BMSM (Crosby \& Mena, 2017; Malebranche, Gvetadze, Millett, \& Sutton, 2012; Smith, 2012). Thus, sex work interventions should address barriers to condom use that may be particularly salient to BMSM-SW, such as client insistence, gender role conflict, race, and homophobia.

Given the increased STI burden in this analysis, interventions of BMSM-SW should encourage urine and rectal STI tests at frequent intervals (e.g., every three months or more frequently). While STI screening may be standard among MSM using preexposure prophylaxis (PrEP), a more comprehensive approach should be the quarterly screening of BMSM-SW regardless of PrEP use, given that PrEP uptake among Black MSM has been slower than among other MSM (Jenness et al., 2018). Efforts to address STI infections among MSM engaged in sex work depend on the identification and referral of MSM to programs for sex work education and harm reduction. Therefore, additional inquiries into the fidelity of providers initiating conversations with MSM about sex work may also prove valuable.

It is necessary to design, implement, and evaluate sexual and psychosocial health interventions for Black MSM engaged in sex work. Given the nature of exchange sex and other forms of sex work, additional research is needed to better understand biobehavioral HIV prevention tool (PrEP/
PEP) access and use for seronegative BMSM-SW and HIV care outcomes for BMSM-SW who are living with HIV. There is a strong need for prospective cohort studies of young MSM who may be at risk for involvement in sex work to better elucidate the temporal relationships between sex work engagement, psychosocial health conditions, and STI incidence. Additionally, because the HIV/STI risks of male sex work may vary along the various online and physical venues where MSW and clients meet (Minichiello, Scott, \& Callander, 2015; Schrimshaw et al., 2017), further research into the different types of sex work engagement will be important to properly design harm reduction interventions. Implications of the usefulness of interventions that not only support biomedical advances, but that attenuate the impact of discrimination and violence are paramount for BMSM-SW.

Acknowledgements We thank the Center for Black Equity and local Black Pride organizations for partnering with us to implement POWER, the community-based organizations who performed onsite HIV testing on the study's behalf, the thousands of study participants who volunteered their time to contribute to this research, and members of the POWER Study Team who made data collection possible. The local Black Pride organizations are as follows: D.C. Black Pride, Detroit's Hotter than July, Houston Splash, In the Life Atlanta, Memphis Black Pride, and Philadelphia Black Pride. The community-based organizations who performed onsite HIV testing are as follows: Atlanta: AID Atlanta, AIDS Health Care Foundation, NAESM; Detroit: Community Health Awareness Group, Horizons Project, Unified; Houston: Avenue 360, Houston AIDS Foundation, Positive Efforts; Memphis: Friends for Life; Philadelphia: Access Matters, Philadelphia FIGHT; Washington, D.C.: Us Helping Us. An earlier version of this manuscript was presented at the 2018 International Academy of Sex Research meeting (Madrid, Spain).

Funding This study was partially supported by National Institutes of Health Grant R01NR013865 and analysis was partially supported by NIMH training Grant 5-T32MH094174-09.

\section{Compliance with Ethical Standards}

Conflict of interest There are no conflicts of interest to report for any of this paper's contributing authors.

Ethical Approval All study procedures were approved by the University of Pittsburgh Institutional Review Board.

Informed Consent Informed consent was obtained for all enrolled participants. All study procedures were approved by the University of Pittsburgh Institutional Review Board.

\section{Appendix 1}

See Table 5. 
Table 5 Direct, indirect, and total effects of relationships between past-year sex work (predictor), syndemic psychosocial conditions (mediator), and past-year STI diagnoses (outcome) among Black MSM in POWER, 2014-2017 $(n=4421)$

\begin{tabular}{|c|c|c|c|c|c|c|}
\hline Outcome and mediator paths & Beta coefficient & SE & $z$ & $p$ value & $95 \% \mathrm{LI}$ & $95 \%$ UI \\
\hline \multicolumn{7}{|c|}{ Factor loading on STI burden (latent): direct effects } \\
\hline Chlamydia & 1 (constrained) & - & - & - & - & - \\
\hline Syphilis & 0.8063532 & 0.02 & 35.26 & $<.0001$ & 0.76 & 0.85 \\
\hline Other STI & 0.73 & 0.02 & 36.08 & $<.0001$ & 0.69 & 0.77 \\
\hline Gonorrhea & 0.99 & 0.02 & 39.86 & $<.0001$ & 0.94 & 1.04 \\
\hline \multicolumn{7}{|c|}{ Factor loading on psychosocial syndemic (latent): direct effects } \\
\hline Intimate partner violence & 1 (constrained) & - & - & - & - & - \\
\hline Physical assault & 0.99 & 0.02 & 40.8 & $<.0001$ & 0.95 & 1.04 \\
\hline Polydrug use & 0.24 & 0.02 & 14.54 & $<.0001$ & 0.21 & 0.28 \\
\hline Depression symptoms & 0.42 & 0.02 & 17.29 & $<.0001$ & 0.37 & 0.47 \\
\hline \multicolumn{7}{|l|}{ STI burden (latent): direct effects } \\
\hline Psychosocial syndemics (latent) & 0.21 & 0.02 & 13.78 & $<.001$ & 0.18 & 0.25 \\
\hline Past-year sex work & 0.04 & 0.02 & 2.12 & .034 & 0.00 & 0.08 \\
\hline Hispanic/Latino ethnicity & 0.13 & 0.02 & 5.74 & $<.001$ & 0.08 & 0.17 \\
\hline Year & 0.01 & 0.00 & 1.42 & .155 & 0.00 & 0.01 \\
\hline Age 40 or older & -0.02 & 0.01 & -1.59 & .111 & -0.04 & 0.00 \\
\hline City sampled & 0.00 & 0.00 & -0.26 & .792 & -0.01 & 0.00 \\
\hline Low-income status & 0.00 & 0.01 & 0.07 & .945 & -0.02 & 0.02 \\
\hline Past-year sex partners, number & 0.00 & 0.00 & 6.24 & $<.001$ & 0.00 & 0.00 \\
\hline HIV status & 0.03 & 0.01 & 3.64 & $<.001$ & 0.01 & 0.05 \\
\hline Bisexual behavior, past year & 0.03 & 0.01 & 2.61 & .009 & 0.01 & 0.05 \\
\hline \multicolumn{7}{|c|}{ Psychosocial syndemic conditions (latent): direct effects } \\
\hline Past-year sex work & 0.33 & 0.02 & 13.94 & $<.001$ & 0.28 & 0.37 \\
\hline Hispanic/Latino ethnicity & 0.05 & 0.03 & 1.73 & .084 & -0.01 & 0.11 \\
\hline Year & -0.01 & 0.01 & -2.25 & .025 & -0.02 & 0.00 \\
\hline Age 40 or older & -0.06 & 0.01 & -4.01 & $<.001$ & -0.09 & -0.03 \\
\hline City sampled & 0.00 & 0.00 & -0.6 & .551 & -0.01 & 0.00 \\
\hline Low-income status & 0.08 & 0.01 & 5.92 & $<.001$ & 0.05 & 0.10 \\
\hline Past-year sex partners, number & 0.00 & 0.00 & 2.69 & .007 & 0.00 & 0.00 \\
\hline HIV status & 0.05 & 0.01 & 4.12 & $<.001$ & 0.02 & 0.07 \\
\hline Bisexual behavior, past year & 0.09 & 0.01 & 6.37 & $<.001$ & 0.06 & 0.12 \\
\hline \multicolumn{7}{|c|}{ STI burden (latent): indirect effects, adjusted for mediator } \\
\hline Past-year sex work & 0.07 & 0.01 & 9.9 & $<.001$ & 0.06 & 0.08 \\
\hline Hispanic/Latino ethnicity & 0.01 & 0.01 & 1.72 & .086 & 0.00 & 0.02 \\
\hline Year & 0.00 & 0.00 & -2.22 & .026 & 0.00 & 0.00 \\
\hline Age 40 or older & -0.01 & 0.00 & -3.86 & $<.001$ & -0.02 & -0.01 \\
\hline City sampled & 0.00 & 0.00 & -0.6 & .551 & 0.00 & 0.00 \\
\hline Low-income status & 0.02 & 0.00 & 5.46 & $<.001$ & 0.01 & 0.02 \\
\hline Past-year sex partners, number & 0.00 & 0.00 & 2.64 & .008 & 0.00 & 0.00 \\
\hline HIV status & 0.01 & 0.00 & 3.96 & $<.001$ & 0.00 & 0.01 \\
\hline Bisexual behavior, past year & 0.02 & 0.00 & 5.81 & $<.001$ & 0.01 & 0.03 \\
\hline \multicolumn{7}{|l|}{ STI burden (latent): total effects } \\
\hline Psychosocial syndemics (latent) & 0.21 & 0.02 & 13.78 & $<.001$ & 0.18 & 0.25 \\
\hline Past-year sex work & 0.11 & 0.02 & 5.96 & $<.001$ & 0.07 & 0.15 \\
\hline Hispanic/Latino ethnicity & 0.14 & 0.02 & 6.07 & $<.001$ & 0.09 & 0.18 \\
\hline Year & 0.00 & 0.00 & 0.78 & .437 & 0.00 & 0.01 \\
\hline Age 40 or older & -0.03 & 0.01 & -2.65 & .008 & -0.05 & -0.01 \\
\hline City sampled & 0.00 & 0.00 & -0.42 & .675 & -0.01 & 0.00 \\
\hline Low-income status & 0.02 & 0.01 & 1.68 & .093 & 0.00 & 0.04 \\
\hline Past-year sex partners, number & 0.00 & 0.00 & 6.82 & $<.001$ & 0.00 & 0.00 \\
\hline HIV status & 0.04 & 0.01 & 4.68 & $<.001$ & 0.02 & 0.06 \\
\hline
\end{tabular}


Table 5 (continued)

\begin{tabular}{lllrrrr}
\hline Outcome and mediator paths & Beta coefficient & SE & $z$ & $p$ value & 95\% LI & 95\% UI \\
\hline Bisexual behavior, past year & 0.05 & 0.01 & 4.3 & $<.001$ & 0.03 & 0.07 \\
Psychosocial syndemic conditions & (latent): total effects & & & & & \\
Past-year sex work & 0.33 & 0.02 & 13.94 & $<.001$ & 0.28 & 0.37 \\
Hispanic/Latino ethnicity & 0.05 & 0.03 & 1.73 & .084 & -0.01 & 0.11 \\
Year & -0.01 & 0.01 & -2.25 & .025 & -0.02 & 0.00 \\
Age 40 or older & -0.06 & 0.01 & -4.01 & $<.001$ & -0.09 & -0.03 \\
City sampled & 0.00 & 0.00 & -0.6 & .551 & -0.01 & 0.00 \\
Low-income status & 0.08 & 0.01 & 5.92 & $<.001$ & 0.05 & 0.10 \\
Past-year sex partners, number & 0.00 & 0.00 & 2.69 & .007 & 0.00 & 0.00 \\
HIV status & 0.05 & 0.01 & 4.12 & $<.001$ & 0.02 & 0.07 \\
Bisexual behavior, past year & 0.09 & 0.01 & 6.37 & $<.001$ & 0.06 & 0.12 \\
\hline
\end{tabular}

\section{Appendix 2}

\section{See Figure 2.}

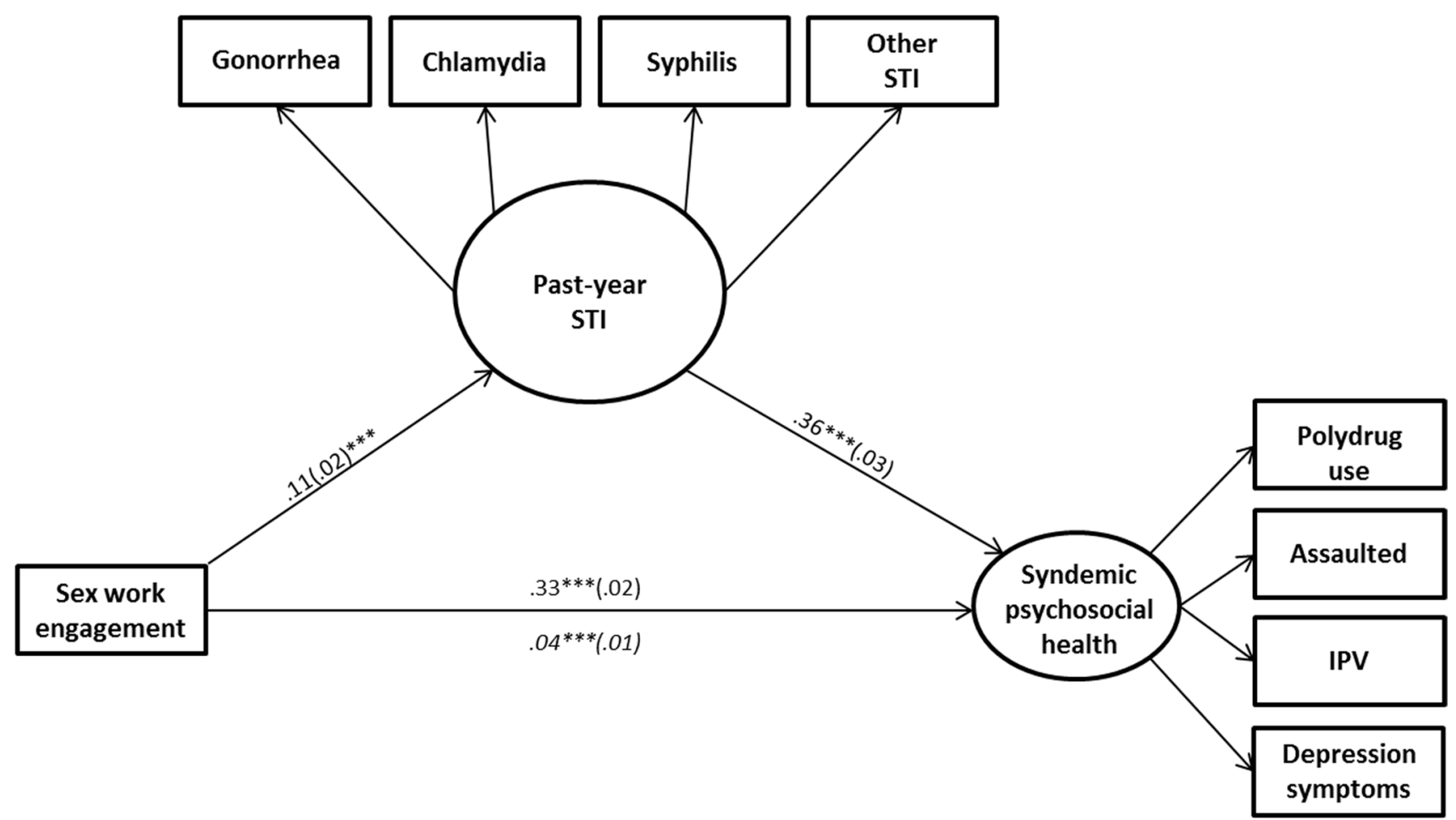

Fig. 2 Structural equation model showing total and indirect effects pathways between past-year sex work, syndemic psychosocial conditions, and STI burden among sexually active Black men in the POWER study, 2014-2017. *** $p<.001$. Path coefficients and standard errors (parenthesized) are shown for total effects pathways. Italicized path coefficients and standard errors (parenthesized) are shown for indirect effects pathways. Model adjusted for year, city, income, bisexual behavior, Hispanic ethnicity, HIV positive status, bisexual behavior, and age $\geq 40$. Covariate paths are suppressed for interpretability. Fit statistics are as follows: $\mathrm{SRMR}=0.034$; $\mathrm{RMSEA}=0.049$ (95\% CI 0.046, 0.052); CFI $=0.928 ; \mathrm{TLI}=0.902$ 


\section{Appendix 3}

See Table 6

Table 6 Total and indirect path coefficients and standard errors in pathways between sex work engagement and psychosocial health syndemic among Black MSM in the POWER study, 2014-2017 ( $n=4421)$

\begin{tabular}{|c|c|c|c|c|c|}
\hline \multirow[t]{2}{*}{ Predictors and covariates } & \multicolumn{2}{|l|}{ Total effects } & \multicolumn{3}{|c|}{ Indirect effects in model adjusting for mediator (latent STI burden) } \\
\hline & $\beta(\operatorname{SE} \beta)$ & $p$ & $\beta(\operatorname{SE} \beta)$ & $p$ & $\begin{array}{l}\text { Proportion of effect mediated by STI } \\
\text { burden }(\% \text { and } 95 \% \mathrm{CI})\end{array}$ \\
\hline Past-year sex work engagement & $0.33(0.02)$ & $<.001$ & $0.04(0.01)$ & $<.001$ & $11.9 \%(8.9 \%, 14.2 \%)$ \\
\hline
\end{tabular}

Model adjusted for year, city, income, bisexual behavior, Hispanic ethnicity, HIV positive status, bisexual behavior, number of past-year sexual partners, and age $\geq 40$. SRMR $=0.034$; RMSEA $=0.049$ (95\% CI 0.046, 0.052); CFI $=0.928 ; \mathrm{TLI}=0.902$

Beta coefficients and respective standard errors reported

\section{References}

Andresen, E. M., Malmgren, J. A., Carter, W. B., \& Patrick, D. L. (1994). Screening for depression in well older adults: Evaluation of a short form of the CES-D. American Journal of Preventive Medicine, 10(2), 77-84.

Balaji, A. B., Bowles, K. E., Hess, K. L., Smith, J. C., Paz-Bailey, G., \& Group, N. S. (2017). Association between enacted stigma and HIV-related risk behavior among MSM, National HIV Behavioral Surveillance System, 2011. AIDS and Behavior, 21(1), 227-237.

Baral, S. D., Friedman, M. R., Geibel, S., Rebe, K., Bozhinov, B., Diouf, D., ... Cáceres, C. F. (2015). Male sex workers: Practices, contexts, and vulnerabilities for HIV acquisition and transmission. The Lancet, 385(9964), 260-273.

Bauermeister, J. A., Eaton, L., Meanley, S., Pingel, E. S., \& Partnership, U. (2017). Transactional sex with regular and casual partners among young men who have sex with men in the Detroit metro area. American Journal of Men's Health, 11(3), 498-507.

Bauermeister, J. A., Eaton, L., \& Stephenson, R. (2016). A multilevel analysis of neighborhood socioeconomic disadvantage and transactional sex with casual partners among young men who have sex with men living in metro Detroit. Behavioral Medicine, 42(3), 197-204.

Biello, K. B., Colby, D., Closson, E., \& Mimiaga, M. J. (2014). The syndemic condition of psychosocial problems and HIV risk among male sex workers in Ho Chi Minh City, Vietnam. AIDS and Behavior, 18(7), 1264-1271.

Biello, K. B., Oldenburg, C. E., Mitty, J. A., Closson, E. F., Mayer, K. H., Safren, S. A., \& Mimiaga, M. J. (2017). The "Safe Sex" conundrum: Anticipated stigma from sexual partners as a barrier to PrEP use among substance using MSM engaging in transactional sex. AIDS and Behavior, 21(1), 300-306.

Bobashev, G. V., Zule, W. A., Osilla, K. C., Kline, T. L., \& Wechsberg, W. M. (2009). Transactional sex among men and women in the south at high risk for HIV and other STIs. Journal of Urban Health, $86(1), 32-47$.

Bukowski, L. A., Chandler, C. J., Creasy, S. L., Matthews, D. D., Friedman, M. R., \& Stall, R. D. (2018). Characterizing the HIV care continuum and identifying barriers and facilitators to HIV diagnosis and viral suppression among black transgender women in the United States. Journal of Acquired Immune Deficiency Syndromes, 79(4), 413-420.

Crosby, R. A., \& Mena, L. (2017). Correlates of enhanced sexual pleasure from condom use: A study of young black men who have sex with men in the United States. AIDS and Behavior, 21(5), 1491-1496.

Dilley, J., McFarland, W., \& Kellogg, T. (2000). Use of a unique testing code among anonymous testers to track repeat tests, estimate HIV incidence, and identify risk factors for HIV seroconversion [Abstract MoPpD1132]. Paper presented at the International AIDS Conference, Durban, South Africa.

dos Ramos Farías, M. S., Garcia, M. N., Reynaga, E., Romero, M., Vaulet, M. L. G., Fermepín, M. R., ... González, J. V. (2011). First report on sexually transmitted infections among trans (male to female transvestites, transsexuals, or transgender) and male sex workers in Argentina: High HIV, HPV, HBV, and syphilis prevalence. International Journal of Infectious Diseases, 15(9), e635-e640.

Dyer, T. P., Shoptaw, S., Guadamuz, T. E., Plankey, M., Kao, U., Ostrow, D., ... Stall, R. (2012). Application of syndemic theory to black men who have sex with men in the Multicenter AIDS Cohort Study. Journal of Urban Health, 89(4), 697-708.

Dyer, T. V., Turpin, R. E., Stall, R., Khan, M. R., Nelson, L. E., Brewer, R., ... Mayer, K. H. (2020). Latent profile analysis of a syndemic of vulnerability factors on incident sexually transmitted infection in a cohort of black men who have sex with men only and black men who have sex with men and women in the HIV prevention trials network 061 study. Sexually Transmitted Diseases, 47(9), 571-579.

Eaton, L. A., Matthews, D. D., Driffin, D. D., Bukowski, L., Wilson, P. A., Stall, R. D., \& POWER Study Team. (2017). A multi-US city assessment of awareness and uptake of pre-exposure prophylaxis (PrEP) for HIV prevention among Black men and transgender women who have sex with men. Prevention Science, 18(5), 505-516.

Edeza, A., Galárraga, O., Santamaria, E. K., Sosa-Rubí, S., Operario, D., \& Biello, K. B. (2020). "I do try to use condoms, but...": knowledge and interest in PrEP among male sex workers in Mexico City. Archives of Sexual Behavior, 49(1), 355-363.

Farmer, P. (2001). Infections and inequalities: The modern plagues. Berkeley: University of California Press.

Friedman, M. R., Bukowski, L., Eaton, L. A., Matthews, D. D., Dyer, T. V., Siconolfi, D., \& Stall, R. (2019). Psychosocial health disparities among black bisexual men in the U.S.: Effects of sexuality nondisclosure and gay community support. Archives of Sexual Behavior, 48(1), 213-224.

Friedman, M. R., Sang, J. M., Bukowski, L. A., Matthews, D. D., Eaton, L. A., Raymond, H. F., \& Stall, R. (2018). HIV care continuum disparities Among black bisexual Men and the mediating effect 
of psychosocial comorbidities. Journal of Acquired Immune Deficiency Syndromes, 77(5), 451-458.

Guadamuz, T. E., Friedman, M. S., Marshal, M. P., Herrick, A. L., Lim, S. H., Wei, C., \& Stall, R. (2013). Health, sexual health, and syndemics: Toward a better approach to STI and HIV preventive interventions for men who have sex with men (MSM) in the United States. In S. O. Aral, K. A. Fenton, \& J. A. Lipshutz (Eds.), The new public health and STD/HIV prevention (pp. 251-272). New York: Springer.

Halkitis, P. N., Kapadia, F., Bub, K. L., Barton, S., Moreira, A. D., \& Stults, C. B. (2015). A longitudinal investigation of syndemic conditions among young gay, bisexual, and other MSM: The P18 Cohort Study. AIDS and Behavior, 19(6), 970-980.

Hammer, G. P., Kellogg, T. A., McFarland, W. C., Wong, E., Louie, B., Williams, I., ... Klausner, J. D. (2003). Low incidence and prevalence of hepatitis $C$ virus infection among sexually active non-intravenous drug-using adults, San Francisco, 1997-2000. Sexually Transmitted Diseases, 30(12), 919-924.

Hernández-Romieu, A. C., Siegler, A. J., Sullivan, P. S., Crosby, R., $\&$ Rosenberg, E. S. (2014). How often do condoms fail? A crosssectional study exploring incomplete use of condoms, condom failures and other condom problems among black and white MSM in southern USA. Sexually Transmitted Infections, 90(8), 602-607.

Jenness, S. M., Maloney, K. M., Smith, D. K., Hoover, K. W., Goodreau, S. M., Rosenberg, E. S., ... Sullivan, P. S. (2018). The PrEP care continuum and racial disparities in HIV incidence among men who have sex with men. BioRxiv, 249540.

Kelley, C. F., Rosenberg, E. S., O’Hara, B. M., Frew, P. M., Sanchez, T., Peterson, J. L., ... Sullivan, P. S. (2012). Measuring population transmission risk for HIV: An alternative metric of exposure risk in men who have sex with men (MSM) in the US. PLoS ONE, 7(12), e53284. https://doi.org/10.1371/journal.pone.0053284.

Kelley, C. F., Vaughan, A. S., Luisi, N., Sanchez, T. H., Salazar, L. F., Frew, P. M., ... Rosenberg, E. S. (2015). The effect of high rates of bacterial sexually transmitted infections on HIV incidence in a cohort of black and white men who have sex with men in Atlanta, Georgia. AIDS Research and Human Retroviruses, 31(6), 587-592.

Koken, J., Bimbi, D. S., \& Parsons, J. (2010). Male and female escorts: A comparative analysis. In R. Weitzer (Ed.), Sex for sale: Prostitution, pornography, and the sex industry (pp. 205-223). New York: Routledge. https://doi.org/10.4324/9780203872802.

Lanier, Y., \& Sutton, M. Y. (2013). Reframing the context of preventive health care services and prevention of HIV and other sexually transmitted infections for young men: New opportunities to reduce racial/ethnic sexual health disparities. American Journal of Public Health, 103(2), 262-269.

Malebranche, D. J., Gvetadze, R., Millett, G. A., \& Sutton, M. Y. (2012). The relationship between gender role conflict and condom use among Black MSM. AIDS and Behavior, 16(7), 2051-2061.

Matthews, D. D., Herrick, A., Coulter, R. W., Friedman, M. R., Mills, T. C., Eaton, L. A., ... POWER Study Team. (2016). Running backwards: Consequences of current HIV incidence rates for the next generation of black MSM in the United States. AIDS and Behavior, 20(1), 7-16.

Maulsby, C., Millett, G., Lindsey, K., Kelley, R., Johnson, K., Montoya, D., \& Holtgrave, D. (2014). HIV among black men who have sex with men (MSM) in the United States: A review of the literature. AIDS and Behavior, 18(1), 10-25.

Mgbako, O., Park, S. H., Callander, D., Brinker, D. A., Kuhner, C., Carrico, A. W., ... Duncan, D. T. (2019). Transactional sex, condomless anal sex, and HIV risk among men who have sex with men. International Journal of STD and AIDS, 30(8), 795-801.

Mimiaga, M. J., Reisner, S. L., Tinsley, J. P., Mayer, K. H., \& Safren, S. A. (2009). Street workers and internet escorts: Contextual and psychosocial factors surrounding HIV risk behavior among men who engage in sex work with other men. Journal of Urban Health, 86(1), 54-66.

Minichiello, V., Scott, J., \& Callander, D. (2013). New pleasures and old dangers: Reinventing male sex work. Journal of Sex Research, 50(3-4), 263-275.

Minichiello, V., Scott, J., \& Callander, D. (2015). A new public health context to understand male sex work. BMC Public Health, 15(1), 282. https://doi.org/10.1186/s12889-015-1498-7.

Muraguri, N., Tun, W., Okal, J., Broz, D., Raymond, H. F., Kellogg, T., ... Kaiser, R. (2015). HIV and STI prevalence and risk factors among male sex workers and other men who have sex with men in Nairobi, Kenya. Journal of Acquired Immune Deficiency Syndromes, 68(1), 91-96.

Mustanski, B., Phillips, G., Ryan, D. T., Swann, G., Kuhns, L., \& Garofalo, R. (2017). Prospective effects of a syndemic on HIV and STI incidence and risk behaviors in a cohort of young men who have sex with men. AIDS and Behavior, 21(3), 845-857.

Nerlander, L. M., Hess, K. L., Sionean, C., Rose, C. E., Thorson, A., Broz, D., \& Paz-Bailey, G. (2017). Exchange sex and HIV infection among men who have sex with men: 20 US cities, 2011. AIDS and Behavior, 21(8), 2283-2294.

Parsons, J. T., Grov, C., \& Golub, S. A. (2012). Sexual compulsivity, co-occurring psychosocial health problems, and HIV risk among gay and bisexual men: Further evidence of a syndemic. American Journal of Public Health, 102(1), 156-162.

Patel, V. V., Masyukova, M., Sutton, D., \& Horvath, K. J. (2016). Social media use and HIV-related risk behaviors in young black and Latino gay and bi men and transgender individuals in New York City: Implications for online interventions. Journal of Urban Health, 93(2), 388-399.

Philbin, M. M., Kinnard, E. N., Tanner, A. E., Ware, S., Chambers, B. D., Ma, A., \& Fortenberry, J. D. (2018). The association between incarceration and transactional sex among HIV-infected young men who have sex with men in the United States. Journal of Urban Health, 95(4), 576-583.

Raymond, H. F., \& McFarland, W. (2009). Racial mixing and HIV risk among men who have sex with men. AIDS and Behavior, 13(4), 630-637.

Schrimshaw, E. W., Siegel, K., \& Meunier, É. (2017). Venues where male sex workers meet partners: The emergence of gay hookup apps and web sites. American Journal of Public Health, 107(12), 1866.

Singer, M. C., Bulled, N., Ostrach, B., \& Mendenhall, E. (2017). Syndemics and the biosocial conception of health. The Lancet, 389(10072), 941-950.

Singer, M. C., Erickson, P. I., Badiane, L., Diaz, R., Ortiz, D., Abraham, T., \& Nicolaysen, A. M. (2006). Syndemics, sex and the city: Understanding sexually transmitted diseases in social and cultural context. Social Science and Medicine, 63(8), 2010-2021.

Smith, W. P. (2012). Exploring dimensions of racism, homophobia, and social network as concomitant predictors of condom use in a sample of Black MSM. Journal of Gay \& Lesbian Social Services, 24(4), 417-445.

Stall, R., Friedman, M., \& Catania, J. A. (2008). Interacting epidemics and gay men's health: A theory of syndemic production among urban gay men. In R. J. Wolitski, R. Stall, \& R. O. Valdiserri (Eds.), Unequal opportunity: Health disparities affecting gay and bisexual men in the United States (pp. 251-274). New York: Oxford University Press.

Stall, R., Mills, T. C., Williamson, J., Hart, T., Greenwood, G., Paul, J., ... Catania, J. A. (2003). Association of co-occurring psychosocial health problems and increased vulnerability to HIV/AIDS among urban men who have sex with men. American Journal of Public Health, 93(6), 1866-1867.

Sullivan, P. S., Peterson, J., Rosenberg, E. S., Kelley, C. F., Cooper, H., Vaughan, A., ... Del Rio, C. (2014). Understanding racial HIV/STI 
disparities in black and white men who have sex with men: A multilevel approach. PLOS ONE, 9(3), e90514. https://doi.org/10.1371/ journal.pone.0090514.

Tobin, K. E., Yang, C., King, K., Latkin, C. A., \& Curriero, F. C. (2016). Associations between drug and alcohol use patterns and sexual risk in a sample of African American men who have sex with men. AIDS and Behavior, 20(3), 590-599.

Tsai, A. C., \& Burns, B. F. (2015). Syndemics of psychosocial problems and HIV risk: A systematic review of empirical tests of the disease interaction concept. Social Science and Medicine, 139, 26-35.

Underhill, K., Morrow, K. M., Colleran, C. M., Holcomb, R., Operario, D., Calabrese, S. K., ... Mayer, K. H. (2014). Access to healthcare, HIV/STI testing, and preferred pre-exposure prophylaxis providers among men who have sex with men and men who engage in streetbased sex work in the US. PLoS ONE, 9(11), e112425. https://doi. org/10.1371/journal.pone.0112425.

Valente, P. K., Mantell, J. E., Masvawure, T. B., Tocco, J. U., Restar, A. J., Gichangi, P., ... Sandfort, T. G. (2020). "I couldn't afford to resist": Condom negotiations between male sex workers and male clients in Mombasa, Kenya. AIDS and Behavior, 24(3), 925-937.

Walters, S. M., Braksmajer, A., Coston, B., Yoon, I., Grov, C., Downing, M. J., ... Hirshfield, S. (2020). A syndemic model of exchange sex among hiv-positive men who have sex with men. Archives of Sexual Behavior, 49(6), 1965-1978.

Wilson, P. A., Nanin, J., Amesty, S., Wallace, S., Cherenack, E. M., \& Fullilove, R. (2014). Using syndemic theory to understand vulnerability to HIV infection among Black and Latino men in New York City. Journal of Urban Health, 91(5), 983-998.

Wong, F., Huang, Z., He, N., Smith, B., Ding, Y., Fu, C., \& Young, D. (2008). HIV risks among gay-and non-gay-identified migrant money boys in Shanghai, China. AIDS Care, 20(2), 170-180.

Publisher's Note Springer Nature remains neutral with regard to jurisdictional claims in published maps and institutional affiliations. 